\title{
La formación de Posgrado en Educación en el Ecuador
}

\author{
Postgraduate studies \\ in Education in Ecuador
}

Eduardo Fabara

Universidad Andina Simón Bolívar, Quito - Ecuador eduardo_fabara2001@yahoo.com

Recibido: 6 de septiembre de 2012

Aceptado: 1 de diciembre de 2012

\section{Resumen}

El presente documento contiene una investigación sobre la situación de la formación de Posgrado en Educación en el Ecuador. El estudio se realiza tomando como base el año 2010, aunque muchos aspectos son analizados considerando la situación de los últimos años.

Esta investigación pone en evidencia los problemas existentes en la formación de Posgrado en Educación, como su falta de planificación y las altas necesidades que tiene el sistema educativo para la formación de especialistas de este nivel.

\section{Palabras clave}

Educación superior, posgrados, educación, especialidad, maestrías, cuarto nivel, formación, investigación, presencial, semipresencial, a distancia.

\begin{abstract}
The following article is an investigation about the situation of postgraduate training in Education in Ecuador. The study was based in the year 2010, although many aspects are analyzed taking into consideration the past of couple years.

This research highlights the existing problems in postgraduate training programs in Education, such as the lack of planning and a high need of the educational system to train specialists at this level.
\end{abstract}

\section{Keywords}

Curriculum, cultural studies, cultural representation, signification struggle. 


\section{Introducción}

La realización de programas de Posgrado en Educación en el Ecuador es una de las necesidades más sentidas para el desarrollo armónico del sistema educativo, puesto que es durante el cuarto nivel o posgrado en el que se forman los especialistas de las diferentes áreas que un país requiere.

Todo sistema educativo espera contar con especialistas que se encarguen de los diferentes aspectos de la planificación, proceso gerencial, diseño y administración curricular, mediación pedagógica, investigación e innovación educativa, y de la evaluación y seguimiento de la enseñanza.

Pero además, deben existir profesionales altamente formados en educación de adultos, educación especial, educación intercultural bilingüe, educación técnica y en otras tantas áreas; es decir, cada una de las modalidades y tipos de educación del sistema debe tener profesionales formados en estos campos para promover su adecuado desarrollo. Estos profesionales son los que diseñan y promueven las políticas públicas, investigan, planifican, ejecutan y evalúan los programas específicos de las diferentes modalidades.

La formación especializada de cada uno de estos profesionales se efectúa en el cuarto nivel, puesto que la atención que demandan estos procesos se logra cuando un especialista tiene previamente una formación de tercer nivel o pregrado, además de la necesaria experiencia de trabajo en el aula y una adecuada preparación especializada en nivel de maestría o doctorado que tienen como base, la investigación científica.

Lamentablemente muchas de las decisiones de políticas públicas sobre el sistema educativo se toman sin contar con las respectivas investigaciones que sustenten acciones previstas, entre otras razones, por falta del personal calificado para este fin. El Ministerio de Educación y varias instancias del ramo, a veces toman decisiones a base de propuestas realizadas por técnicos extranjeros que no siempre conocen la realidad del país. La adopción de estas políticas también obedece a la ausencia de técnicos nacionales en las respectivas áreas (Robalino Campos, 2006). La formación de especialistas a nivel de posgrado es una necesidad estratégica que fortalecería la identidad del sistema educativo nacional.

Oscar Ibarra dice que: "La razón fundamental para la creación de maestrías en educación se sintetiza en la necesidad de formar un profesor capaz de conjugar la docencia con la investigación y de posesionar la investigación como elemento clarificador para la formación de cuadros académicos capaces de responder a los momentos cruciales que vive un país" (Ibarra, 2000).

En este sentido, las universidades nacionales tienen la obligación de atender estos requerimientos para asegurar la existencia del talento humano necesario para el desenvolvimiento del sistema de educación.

\section{Objetivo}

El presente estudio tiene como propósito conocer hasta qué punto las universidades ecuatorianas han atendido necesidades de formación de talento humano de alto nivel, qué tipo de especialistas se están formando y si el sistema educativo ha acogido al personal formado en cursos de Posgrado en Educación.

\section{Metodología}

Para estudiar esta situación se revisaron los Posgrados en Educación, autorizados legalmente por la Secretaría Nacional de Educación Superior, Ciencia, Tecnología e Innovación (SENESCYT), en las diferentes universidades del país, cuya información académica se publica en las páginas web de estos organismos. ${ }^{1}$

1 SENECYT es el órgano de gobierno de administración de la educación superior que reemplazó al Consejo de Educación Superior (CONESUP) en octubre de 2010. 
Con el fin de asegurar la confiabilidad de la información, se visitaron las páginas web de las universidades que tenían autorización para organizar programas de Posgrado en Educación. En algunos casos la información no coincidía porque algunos posgrados no llegaron a funcionar o porque la autorización fue solamente para una promoción, la cual se había ejecutado con anterioridad al año de análisis.

En el estudio correspondiente se identificaron únicamente los posgrados que se encontraban en funcionamiento en el año 2010, de modo que no aparecen los que fueron autorizados con posterioridad.

Se complementó el proceso de estudio mediante cinco entrevistas a profesores, una encuesta a veinte estudiantes de la Maestría en Gerencia Educativa de la Universidad Andina Simón Bolívar, y se mantuvieron conversaciones informales con profesores de otras universidades que tienen cuarto nivel en educación, como la Universidad Central, la Universidad Técnica del Norte y la Pontificia Universidad Católica del Ecuador.

\section{Los Posgrados en Educación}

Se llaman posgrados a los estudios universitarios que se realizan con posterioridad a la obtención del título de pregrado y comprenden maestrías, doctorados y los estudios de investigación posdoctoral. Esta es la última fase de la educación formal y tiene como antecedente obligatorio la titulación de pregrado.

En la literatura internacional se establece que los posgrados pueden estar organizados para cumplir con dos finalidades (Brunner, 1996):

a) Profundizar las áreas de estudio en las que se formó el profesional en el tercer nivel o en el pregrado.

b) Desarrollar una formación especializada en áreas operativas en las que se pueda ejercer algún liderazgo en la profesión, después de haber efectuado investigaciones sobre el tema de estudio.

En el primer caso se trataría, por ejemplo, de un profesor de inglés que pueda seguir el cuarto nivel para profundizar su formación en lingüística o en la enseñanza de una segunda lengua.

En el segundo caso, se trataría de que otro profesor de inglés que pueda estudiar supervisión educativa en el cuarto nivel para asesorar a sus colegas en la enseñanza del Inglés, o estudiar investigación educativa para indagar sobre los aspectos que se relacionan con la lingüística o con la enseñanza de un segundo idioma.

Es decir, existe una diferencia entre los dos casos, la misma que es considerada en el currículo de los posgrados por quiénes diseñan y aprueban los cursos respectivos.

La educación superior a nivel de posgrado tuvo su origen en los países desarrollados a partir de los años sesenta del siglo pasado, puesto que el avance de las ciencias hacía que los conocimientos de profesionales que habían seguido alguna carrera universitaria quedaran obsoletos en pocos años; fue así como las principales universidades del mundo impulsaron programas de posgrado siempre vinculados a procesos investigativos.

Otra de las razones para organizar programas de posgrado fue el desarrollo de la ciencia y la tecnología, especialmente de la tecnología informática, la comunicación y la biotecnología. En las universidades se razonó que la mayor parte de profesiones debía incorporar aquellos cambios que se habían producido a raíz de la investigación científica o la tecnología informática para actualizar al profesional, con el fin de asegurar resultados más confiables, seguros y exactos en los servicios ofrecidos.

La incursión de la sociedad en el conocimiento y la información de las diversas comunidades humanas cambió los esquemas de trabajo de varias profesiones. Además, los líderes de varios países se percataron de que la producción del conocimiento era un factor determinante de crecimiento social y humano. 
A ello se agregaron las exigencias cada vez mayores del mercado ocupacional para que los profesionales universitarios atendieran los pedidos más especializados de usuarios de los variados servicios o productos.

Esto significó también mayor demanda en la continua especialización de los profesionales universitarios, por lo que se multiplicaron las ofertas de posgrado en casi todos los países.

\section{Los programas de Posgrado en Educación en el Ecuador}

Los programas de posgrado en el Ecuador se iniciaron a comienzos de los años noventa, es decir, su desarrollo en el ámbito de la educación superior es relativamente nuevo. Apenas 2.450 docentes de la educación inicial, básica y del bachillerato, tienen registrados títulos de cuarto nivel según las estadísticas del Ministerio de Educación, lo que representa solo el 1,16\% de los docentes que trabajan en el sistema educativo a nivel nacional (SIME, 2011).

En el caso de las universidades, el porcentaje era mayor al $28 \%$, en el año 2010 , pero a todas luces insuficiente para atender una buena formación a nivel superior.

En el país no existen actualmente programas de doctorado en educación, por lo que las ofertas que más han proliferado han sido las de maestría, que por lo general se desarrollan con dos años de formación o 96 créditos, con una duración de 16 horas cada crédito. Con menor intensidad se desarrollan programas de especialización, los cuales se ejecutan con una duración de un año académico o el equivalente a 48 créditos.

En 2010 funcionaron 86 programas de Posgrado en Educación, de los cuales 75 fueron de maestría (representando el 87,21\%) y 11 de especialización (o el 12,79\% restante).

\section{Tabla 1. Niveles de formación en el Posgrado en Educación}

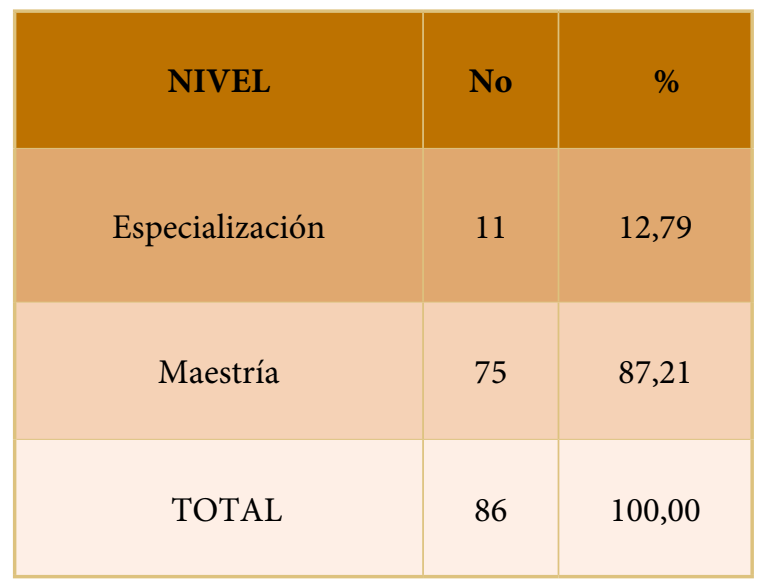

Fuente: SENESCYT. Elaboración: EFG.

Debido a que la nueva Ley Orgánica de Educación Superior no reconoce la especialización como parte del cuarto nivel, se supone que los programas que están funcionando actualmente dejarán de hacerlo cuando finalice el tiempo para el cual fueron autorizados, o serán absorbidos por las maestrías.

Tanto la Ley Orgánica de Educación Superior anterior como la actual, establecen tres modalidades de estudio que son: presencial, semipresencial y a distancia. Los programas de Posgrado en Educación, como la mayor parte de cursos de formación de este nivel, se han desarrollado hasta ahora bajo una modalidad semipresencial, lo que significa que se organiza un sistema modular presencial de un fin de semana cada tres semanas o cada mes, y el resto del tiempo los maestrantes desarrollan sus obligaciones estudiantiles en casa. Esto facilita el trabajo de quienes ejercen su profesión. 
Tabla 2. Modalidad de estudio en el Posgrado en Educación

\begin{tabular}{|c|c|c|}
\hline MODALIDAD & No. & $\%$ \\
\hline Presencial & 13 & 15,12 \\
\hline Semipresencial & 70 & 81,40 \\
\hline A distancia & 3 & 3,49 \\
\hline TOTAL & 86 & 100 \\
\hline
\end{tabular}

Fuente: SENESCYT. Elaboración: EFG

La modalidad semipresencial supera el $81.39 \%$ del total, en tanto que la modalidad presencial alcanza apenas un $15,12 \%$, y la a distancia solo el 3,49\%. Si bien la modalidad semipresencial atiende una necesidad profesional de superación, no todas las áreas académicas son aptas a desarrollarse con este sistema, puesto que el trabajo presencial es absolutamente necesario en algunas ramas del conocimiento, más aún, en aquellas donde las investigaciones deben ejecutarse con la presencia de tutores o a través de actividades grupales de equipos interdisciplinarios que fortalecen la formación individual con su participación diversa.

Para optar por el título correspondiente, los maestrantes deben elaborar y ejecutar un proyecto de investigación sobre uno de los módulos o temas estudiados. Sin embargo, en la actualidad, el aporte que ofrecen estas investigaciones es mínimo, muy pocas son publicadas, unas tantas consultadas por otros maestrantes y la mayoría reposa en los estantes de las bibliotecas de cada universidad, sin dar el aporte investigativo que deberían ofrecer al país, según los estudios realizados por el Convenio Andrés Bello (Aldana, 2005). 
Tabla 3. Universidades con programas de Posgrado en Educación

\begin{tabular}{|c|c|c|}
\hline No. & UNIVERSIDAD & PROGRAMAS \\
\hline 1. & Universidad Central del Ecuador & 8 \\
\hline 2. & Universidad Nacional de Chimborazo & 7 \\
\hline 3. & Universidad Andina Simón Bolívar & 6 \\
\hline 4. & Universidad de Los Andes & 6 \\
\hline 5. & Pontificia Universidad Católica & 6 \\
\hline 6. & Universidad de Guayaquil & 5 \\
\hline 7. & Universidad de Cuenca & 3 \\
\hline 8. & Nacional de Loja & 3 \\
\hline 9. & Universidad Técnica de Ambato & 3 \\
\hline 10. & Universidad Técnica Particular de Loja & 3 \\
\hline 11. & Universidad de Bolívar & 2 \\
\hline 12. & Universidad Técnica de Cotopaxi & 2 \\
\hline 13. & Escuela Superior Politécnica del Litoral & 2 \\
\hline 14. & Universidad Tecnológica Equinoccial & 2 \\
\hline 15. & Universidad Técnica de Machala & 2 \\
\hline 16. & Universidad Técnica de Babahoyo & 2 \\
\hline 17. & Universidad del Azuay & 2 \\
\hline 18. & Universidad San Francisco de Quito & 2 \\
\hline 19. & Universidad Tecnológica América & 2 \\
\hline 20. & Universidad Técnica del Norte & 1 \\
\hline 21. & Universidad Técnica Estatal de Quevedo & 1 \\
\hline 22. & Universidad Politécnica Salesiana & 1 \\
\hline 23. & Escuela Superior Politécnica Ecológica Amazónica & 1 \\
\hline 24. & Escuela Superior Politécnica del Chimborazo & 1 \\
\hline 25. & Escuela Técnica Luis Vargas Torres de Esmeraldas & 1 \\
\hline 26. & Universidad Laica Eloy Alfaro de Manabí & 1 \\
\hline 27. & Universidad Estatal del Sur de Manabí & 1 \\
\hline 28. & Universidad Estatal de Milagro & 1 \\
\hline 29. & Universidad Tecnológica Indoamérica & 1 \\
\hline 30. & Escuela Politécnica del Ejército & 1 \\
\hline 31. & Escuela Politécnica Javeriana & 1 \\
\hline 32. & Universidad Casa Grande & 1 \\
\hline 33. & Universidad Católica Santiago de Guayaquil & 1 \\
\hline 34. & Universidad Metropolitana & 1 \\
\hline 35. & Universidad San Gregorio & 1 \\
\hline 36. & Universidad Tecnológica Empresarial & 1 \\
\hline \multirow[t]{2}{*}{37.} & Universidad San Antonio de Machala & 1 \\
\hline & TOTAL & 86 \\
\hline
\end{tabular}

Fuente: SENESCYT. Elaboración: EFG 
De las 72 universidades existentes en el año 2010, 37 desarrollaron programas de Posgrado en Educación, lo que significa que uno de cada dos centros de educación superior universitaria destinó tiempo y esfuerzos para fortalecer la formación de los docentes ecuatorianos.

Según el número de programas desarrollados se podría hacer una categorización, asignando la mayor participación a seis universidades que son: la Universidad Central, la Universidad Nacional de Chimborazo (sede Riobamba), la Universidad Andina Simón Bolívar, la Universidad Católica del Ecuador, la Universidad de Los Andes (sede Ambato) y la Universidad de Guayaquil, las cuales concentran 38 programas representando un $44 \%$ del total de la oferta posgraduada.

Cabe recalcar que entre estas seis universidades están tres de las más grandes del país: la Universidad de Guayaquil, la Universidad Central del Ecuador y la Universidad Católica del Ecuador. Mientras que la Universidad Andina Simón Bolívar es una entidad exclusivamente de posgrado.

El segundo grupo lo componen 13 universidades que tienen entre dos y tres programas de Posgrado en Educación: la Universidad de Cuenca, la Universidad de Loja, la Técnica de Ambato, la Técnica Particular de Loja, la Estatal de Bolívar, la Técnica de Cotopaxi, la Politécnica del Litoral, la Tecnológica Equinoccial, la Técnica de Machala, la Técnica de Babahoyo, la Universidad del Azuay, la Universidad San Francisco de Quito y la Tecnológica América. Estas universidades ofrecen un total de 30 programas de Posgrado en Educación que equivalen al 35\% de la oferta.

Como se podrá constatar, en este grupo se ubican las universidades estatales de provincia que atienden la costa, y el centro y sur de la sierra. De las 13 universidades, 8 son públicas y 5 privadas.

El tercer grupo está compuesto por 18 universidades que ofertan igual número de programas, es decir, cada universidad tiene su programa de Posgrado en Educación. Los mismos que representan el $21 \%$ de la totalidad. En este grupo se ubican varias escuelas politécnicas y universidades particulares y las restantes son centros de educación superior de varias provincias del país.

La mayor parte de estas últimas universidades no tiene Facultades de Ciencias de la Educación y algunas, ni siquiera programas específicos de educación, abren estos posgrados de manera temporal, de modo que en algunos casos esta formación no guarda armonía con la concentración académica de la universidad. 
Tabla 4. Ubicación de los programas de Posgrado en Educación

\begin{tabular}{|c|c|c|c|}
\hline CIUDAD & PROGRAMAS & CIUDAD & PROGRAMAS \\
\hline Quito & 29 & Latacunga & 2 \\
\hline Ambato & 12 & La Libertad & 2 \\
\hline Guayaquil & 10 & Santo Domingo & 2 \\
\hline Riobamba & 9 & Milagro & 1 \\
\hline Cuenca & 8 & Manta & 1 \\
\hline Loja & 5 & Jipijapa & 1 \\
\hline Ibarra & 4 & Guaranda & 1 \\
\hline Portoviejo & 4 & Babahoyo & 1 \\
\hline Esmeraldas & 3 & Tulcán & 1 \\
\hline Quevedo & 3 & Sangolquí & 103 \\
\hline Machala & 3 & TOTAL & \\
\hline
\end{tabular}

Fuente: SENESCYT. Elaboración: EFG

Geográficamente los Posgrados en Educación se llevan a cabo en 21 ciudades del país. Como se mencionó anteriormente existen 86 programas, sin embargo, varias universidades han organizado el mismo programa en dos o más ciudades, por lo que la oferta crece a 103 opciones educativas de cuarto nivel. De todos ellos, 68 se ubican en cinco ciudades que son: Quito, Ambato, Guayaquil, Riobamba y Cuenca, con 29 de estos concentrados en la ciudad de Quito. Los subsiguientes 28 posgrados se desarrollan en las ciudades de Loja, Ibarra, Portoviejo, Esmeraldas, Quevedo, Machala, La Libertad y Santo Domingo de los Tsáchilas.

Los últimos siete programas de Posgrado en Educación se ejecutan en las ciudades de
Milagro, Manta, Jipijapa, Guaranda, Babahoyo, Tulcán y Sangolquí. Con los cuales se completan las 103 opciones que ofrecieron las universidades en el año 2010.

Llama la atención que ninguna de las capitales provinciales del oriente tenga un programa permanente de formación de docentes en posgrado, como tampoco lo tienen ciudades de la provincia del Cañar, como Azogues o Cañar. A esto habría que agregar que ciudades como Santo Domingo de los Tsáchilas, con una muy alta población, cuentan apenas con dos posgrados. Se debe subrayar que existe una evidente concentración de programas en la ciudad de Quito. 


\section{Gráfico 1}

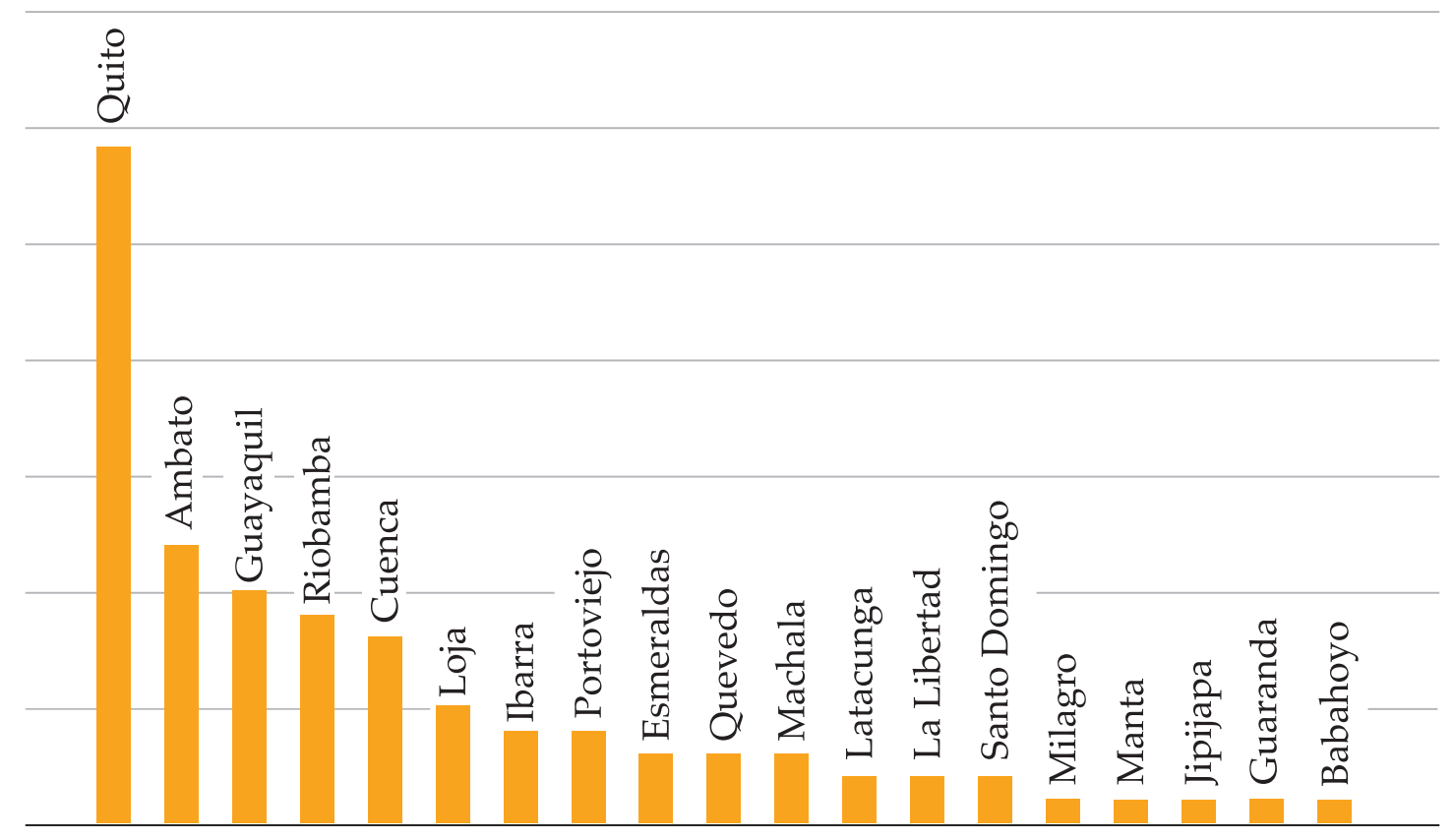

Fuente: SENESCYT. Elaboración EFG.

Lo anterior es una demostración de que no ha existido un proceso de planificación de la educación superior en el país y que la creación de posgrados ha obedecido a factores políticos donde las universidades se han enfocado únicamente en su situación, sin considerar el contexto académico nacional ni las otras ofertas existentes, y donde han influido factores políticos, influencias personales o pago de favores.

En el futuro, la oferta de Posgrados en Educación debe obedecer a factores demográficos y sociales como: el número de docentes graduados de tercer nivel, la proyección de la matrícula estudiantil, el número de planteles educativos con sus respectivas especialidades y los procesos de desarrollo social de las poblaciones atendidas.

\section{Áreas de atención de los Posgrado en Educación}

En cuanto a la naturaleza y las áreas de interés de los programas de posgrado en educación, se pudieron identificar 30 áreas de atención, las cuales tienen que ver con los procesos de profundización de la enseñanza de las diferentes disciplinas científicas, la docencia en los centros de educación superior, el diseño y gestión curricular, la gerencia, planificación y administración de centros, la investigación e innovación educativa, la educación general, la educación infantil, la educación especial, los modelos de evaluación y acreditación y materias con poca relación en el campo del desarrollo educativo, como la prevención y gestión de desastres. 
Tabla 5. Áreas de atención de los Posgrado en Educación

\begin{tabular}{|c|c|}
\hline PROGRAMA DE POSGRADO & CANTIDAD \\
\hline Docencia Universitaria & 10 \\
\hline Educación Superior & 8 \\
\hline Gerencia Educativa & 9 \\
\hline Educación Infantil & 6 \\
\hline Diseño Curricular & 5 \\
\hline Docencia y Currículo & 3 \\
\hline Docencia de las Matemáticas & 7 \\
\hline Lingüística Aplicada, Educación Bilingüe & 7 \\
\hline Educación Especial & 4 \\
\hline Educación & 3 \\
\hline Física Aplicada & 2 \\
\hline Geografía Aplicada & 2 \\
\hline Gestión de Desastres & 2 \\
\hline Investigación e Innovación Pedagógica & 2 \\
\hline Enseñanza de la Historia & 1 \\
\hline Enseñanza del Lenguaje & 1 \\
\hline Enseñanza de la Biología & 1 \\
\hline Enseñanza de la Cultura Física & 1 \\
\hline Pedagogía e Investigación Musical & 1 \\
\hline Desarrollo del Pensamiento & 1 \\
\hline Psicopedagogía & 1 \\
\hline Educación Informática & 1 \\
\hline Educación Inclusiva & 1 \\
\hline Educación para el Desarrollo Sustentable & 1 \\
\hline Planeamiento Educativo & 1 \\
\hline Diseño y Gestión de Proyectos & 1 \\
\hline Proyectos de Desarrollo con Enfoque de Género & 1 \\
\hline Administración Educativa & 1 \\
\hline Evaluación y Acreditación de Educación Superior & 1 \\
\hline Diseño y Evaluación de Modelos Educativos & 1 \\
\hline TOTAL & 86 \\
\hline
\end{tabular}

Fuente: SENESCYT. Elaboración: EFG

Se puede señalar que la profundización de la enseñanza en las diferentes áreas curriculares concentra una cuarta parte de la oferta académica, aunque solamente la docencia para las matemá- 
ticas y el inglés tienen una cantidad aceptable de programas; en el resto de áreas no hay una oferta representativa a nivel nacional.

La otra cuarta parte se destina a formar profesores para la educación superior, entendién- dose que estas universidades tienen interés por formar a sus propios docentes por dos razones: para cumplir con lo establecido en la nueva Ley de Educación Superior y para mejorar la calidad de los aprendizajes.

\section{Tabla 6. Áreas de formación (síntesis)}

\begin{tabular}{|c|c|c|}
\hline N & ÁREAS DE FORMACIÓN EN LOS POSGRADOS & No \\
\hline 1.- & Enseñanza, varias disciplinas & 23 \\
\hline 2.- & Educación Superior & 19 \\
\hline 3.- & Gerencia, Planificación, Administración Educativa & 15 \\
\hline $4 .-$ & Diseño y Gestión Curricular, Investigación e Innovación & 10 \\
\hline 5.- & Educación Infantil & 6 \\
\hline 6.- & Educación Especial & 4 \\
\hline 7.- & Educación General & 3 \\
\hline 8.- & Varias & 6 \\
\hline & TOTAL & 86 \\
\hline
\end{tabular}

Fuente: SENESCYT. Elaboración: EFG

Otra cuarta parte de los programas se destina a la formación de especialistas en: Gerencia Educativa, Administración, Supervisión, Planificación de la Enseñanza, Diseño y Gestión Curricular, y la Investigación e Innovación Educativa. Aquí se denota el interés del sistema por formar a los directivos institucionales, a los técnicos de las universidades, de los planteles educativos y del propio Ministerio de Educación, que tendrán a su cargo los procesos de mejoramiento y cambio del sistema. Así y todo, la oferta es relativamente limitada, considerando la cantidad de autoridades educativas que deben recibir formación especializada.

La cuarta parte final está destinada a la formación en Educación en general, Educación Infantil, Educación Especial y otras. Tampoco aquí se da una oferta representativa, ya que son altos los requerimientos en estos campos, como se demuestra en la siguiente sección. 


\section{Gráfico 2}

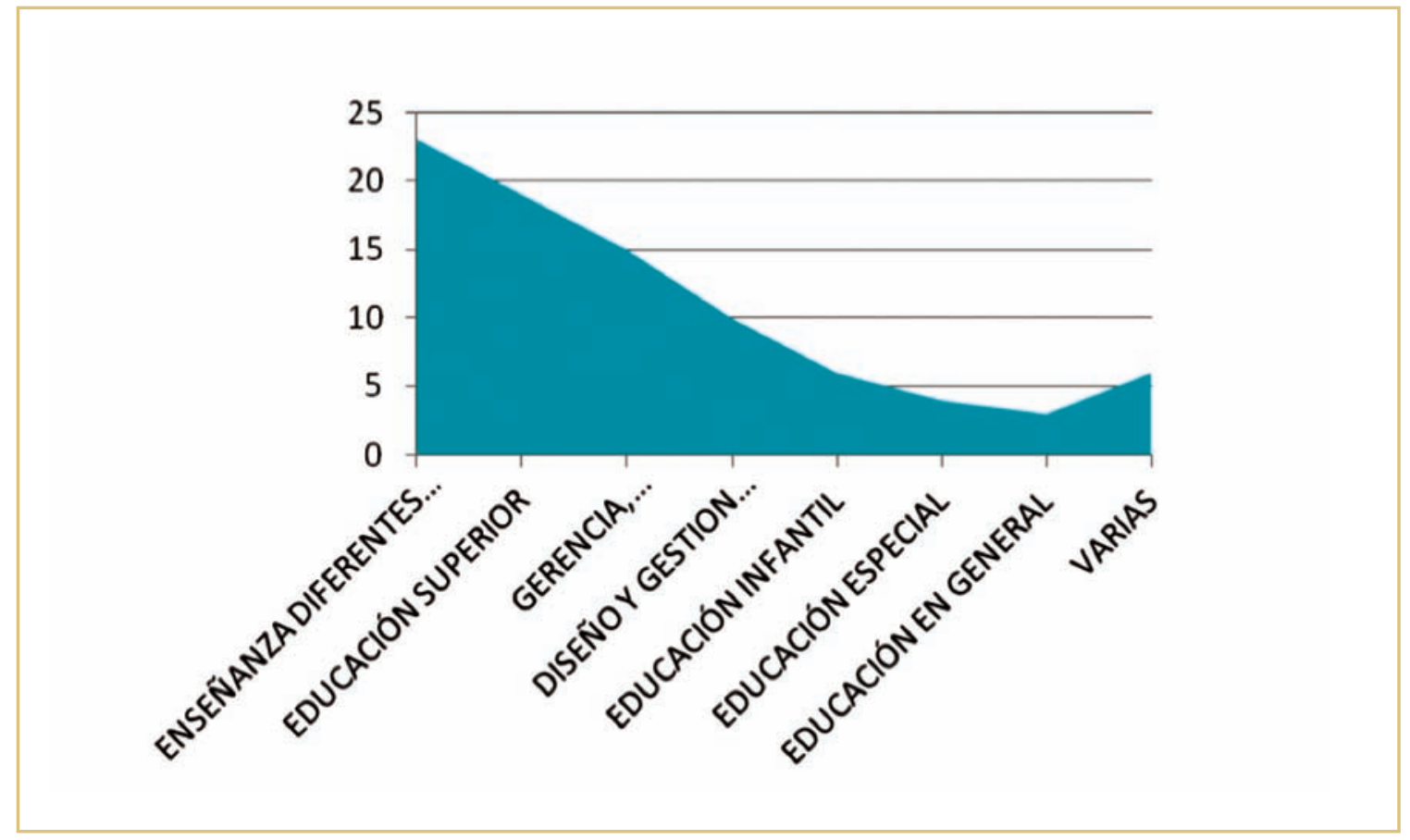

\section{Las necesidades nacionales}

Para reafirmar lo que se mencionó al inicio de este estudio, existen múltiples requerimientos nacionales que no han sido totalmente considerados por los centros de educación superior del país. Se puede señalar que los siguientes grupos necesitan atención urgente:

a) Los docentes de las universidades e institutos superiores, entidades que deben contar con un $50 \%$ de profesores con formación de cuarto nivel, los mismos que deberían poseer títulos de doctorado o maestría en áreas como Docencia Universitaria, Investigación Educativa, Planificación y Currículo, entre otras.

b) Los directivos institucionales de los establecimientos de educación inicial, básica y bachillerato. Lo que significa que los directores de los centros infantiles, de planteles de educación básica, rectores, vicerrectores de colegios y de unidades educativas deberían tener una preparación de posgrado en áreas como Gerencia, Administración, Planificación, Supervisión Educativa y otras ramas afines.

c) Los funcionarios del Ministerio de Educación que pertenecen tanto a la planta central como a las direcciones provinciales y a las diferentes dependencias educativas, entre los cuales se encuentran asesores y auditores educativos (antiguos supervisores), de quienes se espera una alta formación en las respectivas materias de Gerencia de la Educación, Planificación de la Enseñanza, Mediación de los Aprendizajes, Supervisión y Evaluación de los resultados pedagógicos.

d) Los jefes de áreas de las diferentes ramas de la enseñanza y responsables de los proyectos específicos, que tienen la obligación de diri- 
gir y coordinar los programas curriculares de sus respectivos campos de enseñanza y deben tener una mayor preparación en los procesos curriculares, en innovaciones educativas y en la mediación de los aprendizajes.

e) Los directivos institucionales y docentes de los centros educativos especializados en Educación Especial, Educación Inclusiva, Educación Intercultural Bilingüe, Educación de Adultos, Educación Rural, Educación Técnica, Educación Artesanal, Educación Musical, Educación Artística, quienes deben conocer con mayor profundidad el campo de su trabajo específico.

f) Los responsables del desarrollo de los ejes transversales del currículo de la educación básica y bachillerato, como es el caso de la Educación Ambiental, la Educación en Valores, entre otros.

g) Los docentes que lleguen a las dos últimas categorías del nuevo escalafón, quienes, según lo estipula la nueva Ley Orgánica de Educación Intercultural, deben contar con un título de cuarto nivel para ascender de categoría.

En síntesis, si se desea contar en el futuro con un sistema educativo actualizado y que responda a los requerimientos de una educación de calidad, se debe emprender un amplio programa de formación especializada de los docentes nacionales (Aljure Nasser, 1998).

\section{Conclusiones}

Se evidencia que no ha existido una planificación nacional para la apertura de programas de Posgrado en Educación, falta de planificación que se refleja en la escasa atención a las necesidades de formación del talento humano a nivel nacional y en los diferentes campos de desarrollo educativo o en la duplicación indiscriminada de ciertos posgrados, como Gerencia Educativa.

Existe una desigual ubicación geográfica de los programas existentes, los que en crecido número se ubican en la capital de la República, en tanto que existen otras ciudades con menos o ninguna oportunidad de formación en este nivel educativo.

Ninguna universidad ha organizado un programa de Doctorado en Educación que permita potenciar el talento nacional, fortalecer los procesos de alta dirección de la educación y promover la investigación pedagógica. Existen experiencias en otros países en los que varias universidades se asocian para promover una oferta de esta naturaleza o se suscriben convenios con universidades de otros países. Estas experiencias podrían adoptarse en el país para promover la formación de Doctores en Educación a fin de lograr el mejoramiento del sistema educativo.

En la nueva oferta de formación de posgrados se deben considerar las áreas en las que existen pocos o ningún programa de formación en educación.

Los responsables del sistema educativo, las autoridades universitarias, los organismos de crédito educativo y becas, no han establecido sistemas de financiación de estudios de posgrado, especialmente en las áreas en las que no hay especialistas, tal como se puede observar en el Informe del IECE (2010).

Las recomendaciones del autor incluyen:

a) Elaborar y ejecutar un plan de formación de Posgrados en Educación, con la participación de todas las universidades del país, para desarrollarlo en un período de diez años en los que habría que cubrir los déficits existentes en las ramas identificadas.

b) Organizar los Posgrados en Educación considerando las necesidades geográficas del país, para evitar que muchos programas se concentren en pocas ciudades y que otras no cuenten con ellos.

c) Fortalecer los Posgrados en Educación con la modalidad presencial, para asegurar una mayor calidad de egresados del cuarto nivel educativo (Revelo Revelo, 2004). 
d) Organizar un programa de Doctorado en Educación buscando asociar a varias universidades nacionales y contando con la asesoría de una o varias universidades internacionales con experiencia en esta materia, con las cuales se deberían suscribir convenios de participación (González, 2005).

e) Crear redes de instituciones de Posgrado en Educación y de especialistas en las diferentes áreas de cuarto nivel.

f) Organizar un sistema de becas para Posgrados en Educación que se ofrecerían dentro del país $y$ en el exterior, a fin de formar profesionales necesarios para el desarrollo del sistema y para ganar mayor experiencia en este campo.

g) Autorizar el funcionamiento de algunos posgrados en dos o tres promociones con el fin de evitar la saturación de profesionales en algunas áreas en las que se requieren pocos profesionales.

h) Suscribir convenios y acuerdos entre universidades nacionales y del extranjero para implementar programas de profesores invitados, visitantes, pasantías, intercambios de experiencias y de material bibliográfico y didáctico, que permitan optimizar y compartir los recursos existentes en la materia.

\section{Bibliografía}

Aldana, E., et al. (Eds.). 2005. Doctorados para la Integración. Bogotá, Colombia: Convenio Andrés Bello.

Aljure Nasser, E., et al.

1998. Criterios y Procedimientos para la Acreditación de los Programas de Pregrado y Especialización en Educación. Consejo
Nacional de Acreditación (CNA). Bogotá, Colombia: Sistema Nacional de Acreditación. Brunner, J. J. (Ed./Coord.), \& Ferrada Hurtado, R. 2011. Educación Superior en Iberoamérica. Informe 2011. Centro Interuniversitario de Desarrollo (CINDA). Chile: CINDA

Díaz Villa, Mario.

2000. La Formación de Profesores en la Educación Superior Colombiana. Bogotá, Colombia: ICFES. Procesos Editoriales.

Ibarra Russi, O., et al.

2000. Formación de Profesores de la Educación Superior. Bogotá, Colombia: ICFES, Procesos Editoriales.

Fabara Garzón, E.

2011. Estado del Arte de la Formación de Docentes en el Ecuador. Quito, Ecuador: IESALC.

González, L. E.

2005. El Impacto de los Procesos de Autoevaluación en la Educación Superior en Colombia. CINDA-IESALC/UNESCO. Bogotá, Colombia: Convenio Andrés Bello.

Revelo Revelo, J.

2004. Normas, Reflexiones y Realidades sobre la Educación Superior en los Países del Convenio Andrés Bello. Bogotá, Colombia: Convenio Andrés Bello.

Robalino Campos, M., \& Körner, A.

2006. Modelos Innovadores en la Formación Inicial Docente: Una Apuesta por el Cambio. Santiago, Chile: UNESCO.

SENESCYT.

2011. Consulta instituciones de educación superior y carreras. Secretaría Nacional de Educación Superior, Ciencia, Tecnología e Innovación.http://www.senescyt.gob.ec

Sistema de Información del Ministerio de Educación (SIME).

2011. Ministerio de Educación del Ecuador. http://www.educacion.gob.ec 